\title{
Material selection based on ordinal data
}

\begin{abstract}
Ranking and choosing the best material is one of most important stages in material selection process. Using linear assignment method, the multi-criteria decision making (MCDM) approach is proposed in decision-making process to rank the materials for a given engineering component with respect to several criteria. The proposed material selection procedure is relatively simple, and can be a useful approach when material selection problem includes qualitative properties or user-interaction aspects. The suggested approach also can be use for quantitative properties. Three examples are included to demonstrate the suggested method. Result of proposed approach showed good agreement with other methods.
\end{abstract}

Keyword: Weighting and ranking factors $(\mathrm{H})$; Properties of materials (E); Multi-criteria decision making 